\title{
Use of a Benchmark Tracking Assessment to Support Expansion of Buprenorphine for Treatment of Opioid Use Disorder in Primary Care
}

\author{
Andrew L. Sussman, PhD, MCRP, Jennifer N. Crawford, PhD, \\ Heidi Rishel Brakey, MA, Rana S. Alkhafaji, MA, Orrin B. Myers, PhD, \\ Vanessa Jacobsobn, MD, Snehal Bhatt, MD, and Julie Salvador, PhD
}

Introduction: Barriers to the expansion of opioid use disorder (OUD) treatment in primary care using buprenorphine are well documented. Providers require support along a continuum. A systematic tracking framework to enhance provider progress along this continuum is lacking.

Methods: We developed a benchmark tracking assessment (BTA) as part of data collection in a 5-year study to examine the impact of provider participation in an online intervention to support expansion of buprenorphine treatment for OUD in rural primary care. Providers were contacted via phone every 3 months for up to 2 years to track their advancement along the 5 identified key benchmarks and were offered support for any barriers encountered.

Results: Forty-one providers enrolled in the study. Almost half (49\%) did not experience a barrier that prevented them from accomplishing their next benchmark. Of the remaining $51 \%$ of providers, the majority (75\%) experienced barriers early in the training and licensure phases, with most citing lack of time as the main reason.

Conclusion: The BTA offers a feasible approach to identifying challenges along the training to prescription continuum and facilitated targeted support to address barriers. This framework has the potential, with locally contextual adaptations, to guide medication-assisted treatment implementation and training efforts. (J Am Board Fam Med 2021;34:1216-1220.)

Keywords: Access to Health Care, Benchmarking, Buprenorphine, Extensions for Community Healthcare Outcomes (ECHO), Internet-Based Intervention, Medication-Assisted Treatment of Opioid, Opioid-Related Disorders, Opioids, Primary Health Care

\section{Introduction}

Opioid use disorder (OUD) is a major national crisis. ${ }^{1}$ Centers for Disease Control and Prevention (CDC) data show 81,000 drug overdose deaths from May 2019 through April 2020. Exacerbated by COVID-19, this is the highest number of

This article was externally peer reviewed.

Submitted 16 March 2021; revised 17 June 2021; accepted 21 July 2021

From the Department of Family and Community Medicine and University of New Mexico Comprehensive Cancer Center, University of New Mexico Health Sciences Center, Albuquerque (ALS); Department of Psychiatry \& Behavioral Sciences, University of New Mexico Health Sciences Center, Albuquerque (JNC); Clinical and Translational Science Center, University of New Mexico Health Sciences Center, Albuquerque (HRB); Department of Psychiatry \& Behavioral Sciences, University of New Mexico Health Sciences Center, Albuquerque (RSA); Department of Family and Community Medicine, University of New Mexico Health Sciences Center, Albuquerque (OBM); Department of Psychiatry \& overdose deaths ever recorded in a year, and synthetic opioids seem to be the primary driver. ${ }^{1}$ In 2019, New Mexico had the 12th highest drug overdose death rate in the nation, and two thirds of these deaths involved opioids. ${ }^{2}$ Treatment for OUD using Food and Drug Administration (FDA)approved medications including methadone, buprenorphine, or naltrexone is the standard of care. ${ }^{3}$

Recent data show that while the total number of waivered providers has increased, access to

Behavioral Sciences, University of New Mexico Health Sciences Center, Albuquerque (VJ, SB, JS).

Funding: This work was supported by the Agency for Healthcare Research and Quality (1R18HS025345) and the University of New Mexico Clinical and Translational Science Center (CTSC, UL1TR001449).

Conflict of interest: none.

Corresponding author: Andrew L. Sussman, PhD, University of New Mexico Health Sciences Center, Albuquerque (E-mail: asussman@salud.unm.edu). 
medication-assisted treatment (MAT) is still limited, especially in rural areas. Almost one third of rural Americans live in a county without a buprenorphine provider, and many waivered providers do not treat up to currently approved patient capacity limits., ${ }^{4,5}$ Factors contributing to this provider supply problem include low levels of provider confidence in ability to deliver treatment, concerns about buprenorphine diversion, lack of available staff to support clinic workflow and adjunctive psychosocial supports, and burden of buprenorphine training and licensing. ${ }^{6-8}$ The process of acquiring the requisite skills to deliver MAT can be viewed along a continuum with 2 main phases. The first involves a series of training requirements involving completion of DATA waiver training and acquisition of an $\mathrm{x}$-number for licensure. ${ }^{9}$ Subsequently, providers transition to an active prescribing phase during which continued support is necessary to expand patient volume and maximize access.

Given the urgent need to increase MAT access in rural primary care settings, we report here on the implementation and evaluation of a systematic benchmark tracking assessment (BTA) process to support providers along the training-to-prescribing continuum. We developed the BTA to support structured engagement and potential facilitation at key points ("benchmarks") known to either advance or impede provider progress.

\section{Methods}

We developed the BTA for use in an ongoing interventional study aimed at expanding buprenorphine treatment for OUD in rural primary care settings in New Mexico. The study uses the Extensions for Community Healthcare Outcomes (ECHO) model as the primary intervention to deliver training and support to providers. ECHO sessions are held weekly for 12-week cycles and include in-depth didactics on buprenorphine and OUD including prescribing, therapy, and models of clinic functioning (eg, teambased care, screening, billing, and so forth). ${ }^{10}$ In addition, external facilitation support is provided outside the ECHO sessions on request or offered to providers not progressing along the aforementioned buprenorphine implementation steps. Rural primary care providers in New Mexico with no MAT experience or those prescribing buprenorphine for OUD to $\leq 30$ patients within 6 months of enrollment are eligible to participate in the study. Further details of the study have been published elsewhere. ${ }^{11}$

\section{Data Collection}

The BTA consists of 5 key implementation benchmarks grouped into 2 phases. The first 3 benchmarks reflect the training and licensing phase: (1) pre-DATA waiver training, (2) completed DATA waiver but no license, and (3) obtained license but has not prescribed. The second group of benchmarks refers to providers in an active prescribing phase: (4) initiated prescribing to at least 1 patient and (5) expanded treatment to additional patients at each assessment period (see Table 1). At 3-month intervals for a period of 2 years, research staff conduct brief surveys with each participant to assess benchmark progress. If a benchmark is not achieved, the researcher briefly explores barriers, identifies needed support, and offers ECHO clinical team facilitation, which providers may accept or refuse. Providers are also encouraged to request support at any point, regardless of benchmark progress.

\section{Analysis}

We conducted descriptive statistics to show the proportion of providers that missed benchmarks at

Table 1. Benchmark Tracking Assessment Stages, Enrollment Status, and Benchmark Achievement

\begin{tabular}{|c|c|c|c|c|}
\hline $\begin{array}{l}\text { Benchmark Tracking } \\
\text { Assessment Stage }\end{array}$ & MAT Continuum Stage & $\begin{array}{c}\text { Never Missed a } \\
\text { Benchmark }(\mathrm{n}=20) \\
\text { Count }(\%)\end{array}$ & $\begin{array}{c}\text { Missed at Least } 1 \\
\text { Benchmark }(\mathrm{n}=21) \\
\text { Count }(\%)\end{array}$ & $\begin{array}{c}\text { Total } \\
(\mathrm{n}=41) \\
\text { Count }(\%)\end{array}$ \\
\hline 1. Prewaiver & Training and licensing & $10(50.0)$ & $14(66.7)$ & $24(58.5)$ \\
\hline $\begin{array}{l}\text { 2. Between waiver and } \\
\text { license }\end{array}$ & & $2(10.0)$ & $0(0.0)$ & $2(4.9)$ \\
\hline $\begin{array}{l}\text { 3. Between license and } \\
\text { prescribing }\end{array}$ & $2(10.0)$ & $3(14.3)$ & $5(12.2)$ & $0(0.0)$ \\
\hline 4. Prescribing to 1 patient & Actively prescribing & $0(0.0)$ & $0(0.0)$ & $0(0.0)$ \\
\hline $\begin{array}{l}\text { 5. Prescribing to multiple } \\
\text { patients }\end{array}$ & $6(30.0)$ & $4(19.0)$ & $10(24.4)$ & $0(0.0)$ \\
\hline
\end{tabular}

MAT, medication-assisted treatment. 
Table 2. Participant Demographics $(n=41)$

\begin{tabular}{lc}
\hline Gender & $\mathrm{n}(\%)$ \\
\hline Male & $18(43.9)$ \\
Female & $23(56.1)$ \\
Race & \\
American Indian/Alaska Native & $1(2.4)$ \\
Asian & $2(4.9)$ \\
Black, African American & $2(4.9)$ \\
Hawaiian/Pacific Islander & $0(0)$ \\
Non-Hispanic White & $34(82.9)$ \\
Other & $2(4.9)$ \\
Hispanic/non-Hispanic & \\
Hispanic/Latinx & $6(14.6)$ \\
Non-Hispanic/Latinx & $35(85.4)$ \\
Rural & \\
Yes & $41(100.0)$ \\
No & $0(0.0)$ \\
Prescriber Degree & $18(43.9)$ \\
Medical Doctor/Doctor of Osteopathic Medicine & $20(48.8)$ \\
Nurse practitioner & $3(7.3)$ \\
Physician assistant & \\
\hline
\end{tabular}

various stages and at what stage facilitation support was offered or requested. We documented reported barriers through systematic review of provider responses to the brief survey during the BTA. Two trained qualitative researchers documented and organized these responses into general categories.

\section{Results}

A total of 41 rural primary care providers enrolled in the study. Table 2 shows the majority were female $(56.1 \%)$ and non-Hispanic White $(82.9 \%)$. Most participants were either nurse practitioners (48.8\%) or physicians (43.9\%), with physician assistants making up only $7 \%$ of the study population.

\section{Baseline Benchmark Characteristics}

At baseline, most participants had not yet completed training and licensing requirements (75.6\%) to initiate MAT, whereas the remaining $24.4 \%$ entered the study already prescribing to at least 1 patient. Table 1 shows the enrollment benchmark stage stratified by providers that never missed a benchmark and those that missed at least 1 , showing the sample was relatively evenly distributed at baseline by benchmark status.

\section{Benchmark Status and Facilitation Opportunities}

Table 3 characterizes the 103 facilitation opportunities among providers who missed at least 1 benchmark and those who never missed. In this latter group $(\mathrm{n}=20)$, there were 41 facilitation requests from providers to the ECHO team. Most requests (63.4\%) originated while providers were actively prescribing. For all of these requests, support was offered and accepted. Among providers who missed at least 1 benchmark, most facilitation opportunities $(58.1 \%)$ were offered at the early benchmark stages (training and licensing), with providers struggling in the first benchmark stage (prewaiver). A total of 45 facilitation opportunities were offered with missed benchmarks. In contrast to providers requesting support, only slightly over half (56.5\%) of those who missed a benchmark accepted the

Table 3. Facilitation Opportunities at Each Benchmark Stage

\begin{tabular}{|c|c|c|c|c|}
\hline & MAT Continuum Stage & $\begin{array}{c}\text { Never Missed a } \\
\text { Benchmark }(\mathrm{n}=20) \\
\text { Count }(\%)\end{array}$ & $\begin{array}{c}\text { Missed at Least } 1 \\
\text { Benchmark }(\mathrm{n}=21) \\
\text { Count }(\%)\end{array}$ & $\begin{array}{c}\text { Total } \\
(\mathrm{n}=41) \\
\text { Count }(\%)\end{array}$ \\
\hline Number of facilitation opportunities* & & 41 & 62 & 103 \\
\hline Accepted & & $41(100.0)$ & $35(56.5)$ & $76(73.8)$ \\
\hline Average per provider & & 3.7 & 3.0 & 3.2 \\
\hline \multicolumn{5}{|l|}{ Benchmark stage } \\
\hline 1. Prewaiver & Training and licensing & $1(2.4)$ & $22(35.5)$ & $23(22.3)$ \\
\hline 2. Between waiver and license & & $0(0.0)$ & $2(3.2)$ & $2(1.9)$ \\
\hline 3. Between license and prescribing & $14(34.1)$ & $12(19.4)$ & $26(25.2)$ & $0(0.0)$ \\
\hline 4. Prescribing to 1 patient & Actively prescribing & $0(0.0)$ & $1(1.6)$ & $1(1.0)$ \\
\hline 5. Prescribing to multiple patients & $26(63.4)$ & $25(40.3)$ & $51(49.5)$ & $0(0.0)$ \\
\hline Totals & & $41(100)$ & $62(100)$ & $103(100)$ \\
\hline
\end{tabular}

MAT, medication-assisted treatment.

*Offers (ie, after missed benchmark) or requests for facilitation. 
support offered. Facilitation support was offered inperson or virtually, but the vast majority opted for virtual (phone or e-mail consultation), with only 1 provider requesting an in-person meeting.

\section{Barriers to Achieving Benchmarks}

As shown in Table 3, the majority of providers missed a benchmark early in the training and licensing phase, with most not completing the waiver training. When asked about barriers to completing benchmarks, the most commonly cited challenge was lack of time to complete the waiver training ( $\mathrm{n}=12 ; 57 \%$ ). Other providers reported challenges related to the practice setting including leadership's lack of interest in starting or supporting buprenorphine treatment $(\mathrm{n}=7 ; 33 \%)$. Remaining barriers reported were that a provider was a locum and not stable enough at the clinic to start $(\mathrm{n}=1)$, concerns providing treatment to persons with OUD $(n=1)$, and not liking the online ECHO training/support $(n=1)$. As reported previously, providers are highly satisfied with the content and format of the ECHO sessions. ${ }^{10,11}$

\section{Discussion}

This brief report presents use of the BTA to identify providers' challenges across the MAT training and prescribing continuum. Use of the BTA revealed different groupings of barriers among those who missed benchmarks and those who never missed. For the latter, most facilitation requests were from providers actively prescribing to patients and support needs focused primarily on clinical questions pertaining to buprenorphine management. In contrast, among providers who missed at least 1 benchmark, barriers were concentrated in the early benchmark stages with most revolving around time constraints completing DATA waiver training requirements. More than one quarter of providers who missed benchmarks faltered at the waiver stage.

Given the relatively low number of primary care providers implementing buprenorphine treatment for OUD in rural settings, use of a systematic BTA may help to enhance progress by identifying where and why providers stall to direct supportive resources accordingly. Although existing research documents general barriers to buprenorphine treatment expansion among primary care providers, ${ }^{6-8}$ there is limited data on the frequency and types of barriers at discreet stages along the training-to-prescribing continuum.

We developed and implemented the BTA to uncover the multilevel factors-provider, practice, system - that impede or facilitate implementation progress. Providers who express interest in training but are not able to complete licensure may require different types of support compared with those actively prescribing and seeking guidance on expanding patient volumes. While this intervention and facilitation is well suited to support prescribing, behavioral therapy, or clinic process questions once treatment begins via virtual consultation, it is less effective at helping providers overcome practiceand system-level constraints (eg, lack of time, unsupportive medical director) associated with waiver training requirements. These findings have implications in terms of identifying factors that may help to predict types of assistance necessary to best support providers through this process and, at a broader level, may inform current policy debates regarding changing or eliminating DATA waiver training requirements. ${ }^{12-14}$

\section{Limitations}

Patterns of barriers identified among these rural primary care providers in New Mexico may not be generalizable to provider populations in other settings. However, providers cited commonly reported barriers and facilitators to MAT training progress, lending credibility to the application of the BTA in other settings. At the conclusion of this study, we will assess whether other provider demographic and/or practice setting factors providing receipt of training or implementation support may be predictive of benchmark achievement.

\section{Conclusion}

Use of the BTA has enabled a more detailed understanding of barriers to MAT expansion linked to providers at specific phases and supported the delivery of targeted external facilitation. This framework has the potential, with locally contextual adaptations, to guide MAT implementation and training efforts for primary care providers.

We would like to acknowledge the contributions of Laura Rombach, MA, Clark Hardgrave, PA-C, Larissa Maley, PhD, Jesus E. Fuentes, BA, Magdalena M. McWethy, BS, and Justin A. Martinez, BA. 
To see this article online, please go to: http://jabfm.org/content/ 34/6/1216.full.

\section{References}

1. Centers for Disease Control and Prevention; 2020. Overdose deaths accelerating during COVID-19: expanded efforts needed. Available from:https:// www.cdc.gov/media/releases/2020/p1218-overdosedeaths-covid-19.html. Accessed February 25, 2021.

2. New Mexico Department of Health; 2021. Drug overdose in NM factsheet. Available from: https:// www.nmhealth.org/publication/view/marketing/2117/. Accessed May 25, 2021.

3. Mancher M, Leshner AI, editors. Medications for opioid use disorder save lives. Washington (DC): National Academies Press; 2019.

4. Andrilla CHA, Coulthard C, Patterson DG. Prescribing practices of rural physicians waivered to prescribe buprenorphine. Am J Prev Med 2018;54: S208-S214.

5. Ghertner R. U.S. trends in the supply of providers with a waiver to prescribe buprenorphine for opioid use disorder in 2016 and 2018. Drug Alcohol Depend 2019;204:107527.

6. Andrilla CHA, Coulthard C, Larson EH. Barriers rural physicians face prescribing buprenorphine for opioid use disorder. Ann Fam Med 2017;15:359-62.

7. Huhn AS, Dunn KE. Why aren't physicians prescribing more buprenorphine? J Subst Abuse Treat 2017;78:1-7.

8. Hutchinson E, Catlin M, Andrilla HA, Baldwin LM, Rosenblatt RA. Barriers to primary care physicians prescribing buprenorphine. Ann Fam Med 2014;12:128-33.

9. Substance Abuse and Mental Health Services Administration; 2021. Become a buprenorphine waivered practitioner. Available from: https://www. samhsa.gov/medication-assisted-treatment/becomebuprenorphine-waivered-practitioner. Accessed May 28, 2021.

10. Salvador J, Bhatt S, Fowler R, et al. Engagement with project $\mathrm{ECHO}$ to increase medication-assisted treatment in rural primary care. Psychiatr Serv 2019;70:1157-60.

11. Salvador JG, Bhatt SR, Jacobsohn VC, et al. Feasibility and acceptability of an online ECHO intervention to expand access to medications for treatment of opioid use disorder, psychosocial treatments and supports. Subst Abus 2020; 19:1-8.

12. Berk J. To help providers fight the opioid epidemic, "x the X waiver." Health Affairs; 2019. Available from: https://www.healthaffairs.org/do/10.1377/ hblog20190301.79453/full/. Accessed February 25, 2021.

13. Frank JW, Wakeman SE, Gordon AJ. No end to the crisis without an end to the waiver. Subst Abus 2018;39:263-5.

14. Zinsmaster DS, Begin CB. X-waiver changes axed: federal government backtracks on previously announced rescission of waiver requirements. National Law Review; 2021. Available from:https:// www.natlawreview.com/article/x-waiver-changesaxed-federal-government-backtracks-previouslyannounced-rescission. Accessed February 25, 2021. 